\title{
Effect of noise on coupled chaotic systems
}

\author{
Manojit Roy 円 and R. E. Amritkar \& \\ Department of Physics \\ University of Pune \\ Pune-411007, INDIA.
}

\begin{abstract}
Effect of noise in inducing order on various chaotically evolving systems is reviewed, with special emphasis on systems consisting of coupled chaotic elements. In many situations it is observed that the uncoupled elements when driven by identical noise, show synchronization phenomena where chaotic trajectories exponentially converge towards a single noisy trajectory, independent of the initial conditions. In a random neural network, with infinite range coupling, chaos is suppressed due to noise and the system evolves towards a fixed point. Spatiotemporal stochastic resonance phenomenon has been observed in a square array of coupled threshold devices where a temporal characteristic of the system resonates at a given noise strength. In a chaotically evolving coupled map lattice with logistic map as local dynamics and driven by identical noise at each site, we report that the number of structures (a structure is a group of neighbouring lattice sites for whom values of the variable follow certain predefined pattern) follow a power-law decay with the length of the structure. An interesting phenomenon, which we call stochastic coherence, is also reported in which the abundance and lifetimes of these structures show characteristic peaks at some intermediate noise strength.
\end{abstract}

\footnotetext{
${ }^{1}$ email: mroy@physics.unipune.ernet.in

${ }^{2}$ email: rea@physics.unipune.ernet.in
} 


\section{Introduction}

Chaos in natural and human-made systems is a well established fact by now. Systems in diverse disciplines such as population biology [1], physiology [2], hydrodynamics [3], chemical reactions [4], plasma [5], lasers [6], electronics [7], computing networks [8], economic theory [9], social theory etc. have been observed to exhibit rich and unpredictable behaviour of chaos. This behaviour has been identified with the inherent nonlinear nature of the systems rather than external influences.

On the other hand, the very same nonlinearity has been seen to give rise to ordering phenomena [10], e.g., regular formation of cloud patterns, a variety of patterns in hydrodynamic flow, oscillatory patterns in chemical reactions, in behaviour of lasers, pulse propagation in Gunn diode etc. These patterns may be spatial, temporal, or spatiotemporal in nature and their understanding is of very special interest. One particularly interesting ordering phenomenon is the generic existence of different structures in a turbulent fluid [11-18]. They originate and degenerate randomly in space and time. These structures appear in spite of the fact that the fluid is undergoing a turbulent evolution and no clear understanding of this phenomenon is as yet achieved.

Noise has been known to play a detrimental role in many experimental situations. This motivated researchers to develop better techniques and methods to minimize, if not totally remove, the effect of noise and enhance signal-to-noise ratio and hence system performance. Slight amount of noisy perturbation is known to destroy delicate patterns in spatially extended systems.

In light of these facts, interest grew when evidences to the contrary started appearing as regards the effect of noise on the ordering phenomena. Firstly, it has been observed that addition of noise of a given strength to certain nonlinear systems increases the system's response at a particular time scale, thereby improving the signal-to-noise ratio; these findings opened up an entirely new field of research, known as stochastic resonance [19-27]. Secondly, noise has been seen to influence spatial and spatiotemporal behaviour of some nonlinear systems in quite counterintuitive manner. It is observed that noise can trigger, select and sustain patterns in optical systems, fluid dynamical systems etc. [28-32].

Considerable progress has been made to establish connection between 
these pattern forming systems (such as fluid) and nonlinear dynamical systems' theory. A natural next step is to see how does noise influence spatiotemporal evolution of these dynamical systems, with the hope that this kind of study may shed some light on the abovementioned noise-induced features in physical systems. This article presents a review of the work that has been carried out on the effect of noise in inducing order in an otherwise chaotically evolving system, with particular emphasis on systems consisting of coupled chaotically evolving elements.

We have organized the article as follows: Section 2 discusses noise induced ordering phenomena observed in low dimensional uncoupled systems. In Section 3, we review the work on effect of noise on two spatially extended systems, neural network and array of firing elements. Section 4 introduces coupled map lattice (CML), one of the most popular models of nonlinear dynamical systems with spatial extension. In this same section we discuss a recent work that we have carried out on the effect of noise on CML. Section 5 summarises and concludes the article.

\section{Effect of noise on uncoupled systems}

In this section we investigate some examples of noise induced ordering in uncoupled systems.

\subsection{Synchronization}

In some systems different trajectories get synchronized asymptotically to a single noisy trajectory independent of the initial conditions when driven by an identical sequence of noise above certain strength. Here, synchronization should be understood as exponential convergence of the average distance between any two phase space points. This means that synchronization is essentially a nonchaotic phenomenon associated with negative Lyapunov exponent [33], although the asymptotic trajectory can be very random. (Lyapunov exponent $\lambda$ characterizes the rate at which the distance between neighbouring trajectories changes. If $\epsilon_{0}$ is the initial separation between two trajectories, the separation after time $t$ can be written as $\epsilon_{t} \simeq \epsilon_{0} \exp (\lambda t)$. Positive $\lambda$ implies exponential divergence of nearby trajectories and hence chaos. For

multidimensional systems our reference to Lyapunov exponent will always 
mean the maximum of the Lyapunov characteristic exponents.)

In the continuum-time limit such systems can be written as a set of uncoupled Langevin equations

$$
\dot{x}_{i}(t)=F\left(x_{i}\right)+\eta(t),
$$

where $x_{i}$ is a dynamical variable, the index $i$ corresponds to different trajectories i.e. different initial conditions, the (nonlinear) function $F(x)$ governs the dynamics of the system, and $\eta(t)$ is a delta-correlated $\left(\left\langle\eta(t) \eta\left(t^{\prime}\right)\right\rangle=\right.$ $\left.\left\langle\eta^{2}(t)\right\rangle \delta\left(t-t^{\prime}\right)\right)$ noisy driving force imparted at a regular interval small compared to all relevant macroscopic time scales of the system, and is same for all $i$. Eqs. (1) have the synchronization solution $x(t)=x_{i}(t)$. Therefore the question essentially is whether this solution is stable or not for a given range of system parameters. In other words, if an appropriately constructed $\lambda<0$, there is an exponential convergence of trajectories and hence synchronization, and if $\lambda>0$, the evolution is chaotic.

Let us now take up individual cases.

\subsubsection{Particle in newtonian potential}

Fahy and Hamann (FH) [34 have studied a Newtonian particle moving in a smooth multiminima potential $V(x)$ without friction, subjected to the condition that at regular time intervals $\tau$, it is stopped and all its velocity components are reset to random values chosen from a Gaussian distribution. They observed that when an ensemble of such particles with different initial conditions is driven by an identical sequence of random forces, their trajectories asymptotically get synchronized to a single noisy trajectory provided that $\tau$ is less than certain critical value $\tau_{c}$. On the basis of this observation they concluded that for $\tau<\tau_{c}$ the final trajectory of the particles is independent of the initial conditions to any required level of accuracy (the accuracy aspect will have importance later in our discussion); it depends only on the choice of velocities. Thus the trajectory, albeit noisy, is not chaotic for $\tau<\tau_{c}$ because of exponential convergence, and an appropriately defined lyapunov exponent $\lambda$ is negative. They have shown that for any one-dimensional potential $V(x)$ confining the particles to a finite region and for short enough $\tau$, the average rate of contraction $\gamma$ of the distance between two particles initially close together is given by $\gamma=\tau \beta\left\langle(\partial V / \partial x)^{2}\right\rangle / 2 m+O\left(\tau^{2}\right)$, where $\beta=1 / k_{B} T$, 
and $m$ is the mass of the particle (angular brackets denote average with respect to the Boltzmann distribution). The above mentioned synchronization phenomenon is a stronger observation than the well-known statistical phenomenon in which the asymptotic distribution of Brownian trajectories is found to be proportional to the Boltzmann factor $\exp [-\beta V(x)]$, independent of its initial distribution. $\mathrm{FH}$ have also conjectured that this synchronization feature may be generic to all the bounded systems.

\subsubsection{Noisy chaotic systems}

Maritan and Banavar (MB) 35 have considered the effect of noise on the following two chaotic systems. First, they have taken a noisy logistic map with the evolution law

$$
x_{t+1}=4 x_{t}\left(1-x_{t}\right)+\eta_{t},
$$

where $\eta_{t}$ is a uniform-deviate random number chosen from an interval $[-W$, $+W]$ with the constraint that $0<x_{t+1}<1$. Eq. (2) may be considered as an example of the discrete-time version of Eq. (1) with $\Delta t=1$. They found a critical $W_{c}(\approx 0.5)$ such that when a pair of randomly chosen initial conditions is driven by an identical sequence of $\eta_{t}$ with $W>W_{c}$, their asymptotic trajectories become synchronized (within a given accuracy) to a single random trajectory independent of the initial conditions. They have computed the mean squared separation $\bar{d}^{2}$ between the two identically driven trajectories and observed that $\bar{d}^{2}$ falls off for $W>W_{c}$. They offered an intuitive explanation saying that the convergence can occur for logistic map due to the contraction of $d$ whenever sum of the pair of numbers comes close to unity, because the distance evolves as

$$
\left|x_{t+1}(1)-x_{t+1}(2)\right|=4\left|x_{t}(1)-x_{t}(2)\right|\left[1-\left\{x_{t}(1)+x_{t}(2)\right\}\right] .
$$

Since peaks in the invariant density for the logistic map are close to 0 and 1 , $W \geq 1 / 2$ can bring both $x(1)$ and $x(2)$ near $1 / 2$.

The second system considered by MB is the Lorenz system described by

$$
d x / d t=\sigma(x-y), d y / d t=-x z+r x-y, d z / d t=x y-b z,
$$

with $\sigma=10, b=8 / 3$, and $r=28$. The $y$-equation is then evolved in difference form as

$$
y(t+\Delta t)=y(t)+[-x(t) z(t)+r x(t)-y(t)] \Delta t+\eta_{t} W_{l} \sqrt{\Delta t},
$$


where $\eta_{t}$ is again a delta-correlated uniform-deviate random number between $[0,1]$ (it has a nonzero mean unlike in the logistic case) and $W_{l}$ is the amplitude. Note that here addition of noise is unrestricted unlike in the logistic case where boundedness of the phase space constrains noise to depend on the state of the system. For Lorenz system also a threshold value $(\approx 2 / 3)$ for $W_{l}$ was found beyond which synchronization phenomenon was observed for an identically driven system with different initial conditions. It is reported that synchronization does not occur if $\eta_{t}$ with the same amplitude has zero mean.

MB have maintained that although the systems considered above are exhibiting synchronization, their strange attractors are not replaced by topologically simple structures like fixed point or limit cycle.

Pikovsky made a cautionary observation [36] that for all bounded systems there is always a nonzero probability that any two phase space points will come close to within some $\epsilon>0$ with or without noise. In other words after sufficiently long time the two systems will be synchronized because of the finite precision (of the computer) and finiteness of phase space. This type of spurious synchronization will occur even for systems with positive $\lambda$, which is to be distinguished from the physical synchronization which is characterized by negative $\lambda$. The former is unstable against small perturbations because of positive $\lambda$ and so will not be observed in real experiments, whereas the latter is quite stable. Pikovsky found $\lambda$ for noisy logistic system of MB to be positive and concluded that this type of synchronization is a numerical artifact.

MB subsequently noted [37 that whether or not a physical synchronization occurs, identical noise with sufficient strength drastically enhances the probability of 'close encounter' between any two ensemble points.

There has been an attempt [38 to interpret synchronization in terms of the inherent structural instability of the undriven system. These authors maintain that the strange attractor of the undriven chaotic system gets replaced by a stable fixed point under parametric perturbations, resulting in synchronization of any two phase space points and a negative $\lambda$. They showed this for all the three systems mentioned above by treating noise as a perturbation in the parameter and also considered new examples. It was remarked that this phenomenon is not generic and holds for only those systems in which such type of structural instability occurs.

The picture that is emerging out of these findings is that while understanding of the synchronization phenomenon may still remain incomplete, 
there is no doubt about noise playing a crucial role in drastically increasing the chance of close encounter of any two phase space points and thereby bringing down the lyapunov exponents of the otherwise chaotic system.

\subsection{Violation of law of large numbers}

An interesting observation was reported [39] regarding the chaotic evolution of an ensemble of uncoupled maps driven by a parametric noise. The system consists of $N$ local maps

$$
x_{t+1}(i)=F\left(x_{t}(i) ; a_{t}(i)\right) \text {, }
$$

where the nonlinearity parameter $a_{t}(i)$ is subjected to fluctuations in both space and time. The quantity of interest is the mean field $h_{t}$, defined as

$$
h_{t}=\frac{1}{N} \sum_{j=1}^{N} F\left(x_{t}(j)\right) .
$$

For the uncoupled variables $x_{t}(i)$ fluctuating almost independently, $h_{t}$ for large $N$ is expected to obey the law of large numbers, and hence the mean-

square deviation (MSD) $\left(=\left\langle h_{t}^{2}\right\rangle-\left\langle h_{t}\right\rangle^{2}\right)$ should vary as $1 / N$, and converge to a fixed-point value as $N \rightarrow \infty$. It was observed that with the logistic map in chaotic regime, MSD falls off as $\sim 1 / N$ if $a_{t}(i)$ fluctuates in space. On the other hand if $a_{t}(i)=a_{t}$ is independent of $i$ then MSD saturates with $N$ beyond a critical $N=N_{c}$, whose value depends on the strength of the noise. Similar behaviour was observed using other maps like the circle and tent maps. The author claims that an ensemble of uncoupled chaotic maps with spatially uniform parametric fluctuations violates the law of large numbers, irrespective of the details of the map.

\section{Effect of noise on spatially extended sys- tems}

We shall now consider systems which have a spatial extension. Because of increased complexity owing to the largely enhanced phase space dimensions (one mostly talks of infinite dimensional phase spaces) these systems show 
very rich dynamical features. Spatially extended systems are modelled by a set of variables (whose evolution may be governed by discrete-time nonlinear maps like the logistic map, or continuous-time ordinary differential equations such as the oscillator equation) physically coupled to each other in euclidean space.

Let us consider a few cases of such systems and the influence of noise on their evolution.

\subsection{Noise and suppression of chaos in neural network}

Molgedey, Schuchhardt and Schuster (MSS) have investigated the effect of noise on discrete-time evolution of a random neural network with infiniterange interactions 40. The model consists of $N$ analog neurons $\left\{x_{t}(i)\right\}$, $i=1, \cdots, N$, with $-1 \leq x(i) \leq 1$, evolving according to the law

$$
\begin{aligned}
x_{t+1}(i) & =F\left(h_{t}(i)\right), \\
h_{t}(i) & =\sum_{j \neq i} \varepsilon_{i j} x_{t}(j)+\eta_{t}(i),
\end{aligned}
$$

where the function $F(h)$ has the following properties: 1 . it is odd $[F(-h)=$ $-F(h)] ; 2$. it approaches \pm 1 as $h \rightarrow \pm \infty ; 3$. it increases near $h=0$ as $[d F / d h]_{h=0}=g$, where $g>0$ is the gain parameter. The coupling parameters $\varepsilon_{i j}$ are delta-correlated Gaussian random variables with zero mean, $h_{t}(i)$ denotes the internal field of the neuron and $\eta_{t}(i)$ is the external white noise with zero mean and variance $\left\langle\eta_{t}(i) \eta_{\tau}(j)\right\rangle=\sigma^{2} \delta_{i j} \delta_{t \tau}$.

MSS used a dynamical functional approach to reduce the dynamics of the entire system to an equation for an effective single neuron in the thermodynamical limit:

$$
x_{t+1}=F\left(h_{t}\right) \text {, }
$$

with

$$
\left\langle h_{t} h_{\tau}\right\rangle=\sigma^{2} \delta_{t \tau}+\left\langle F\left(h_{t-1}\right) F\left(h_{\tau-1}\right)\right\rangle,
$$

Eq. (8) with Eq. (9) yields the same averaged dynamical properties as for

Eq. (6). One now defines activity of the network as $K_{t}=\left\langle h_{t}^{2}\right\rangle$ which for small $K$ reduces to

$$
K_{t}=\sigma^{2}+g^{2} K_{t-1}+O\left(K_{t-1}^{2}\right) .
$$


In the absence of noise there is a trivial fixed point $K^{*}=0$ for Eq. (10) and is stable for $g<1$. In the presence of noise it has only one stable fixed point in the range $\left[0,1+\sigma^{2}\right]$.

To study the chaotic behaviour of the network a replica of the system with infinitesimally separated initial conditions and with the same noise $\eta_{t}^{1}(i)=$ $\eta_{t}^{2}(i)$ is constructed. Lyapunov exponent for the system is defined as

$$
\lambda=\lim _{\tau \rightarrow \infty} \lim _{x_{t}^{1} \rightarrow x_{t}^{2}} \frac{1}{2 \tau} \log _{2} \frac{\left\langle\left(x_{t+\tau}^{1}-x_{t+\tau}^{2}\right)^{2}\right\rangle}{\left\langle\left(x_{t}^{1}-x_{t}^{2}\right)^{2}\right\rangle} .
$$

Assuming equilibrium $\left(\left\langle\left(h_{t}^{1}\right)^{2}\right\rangle=\left\langle\left(h_{t}^{2}\right)^{2}\right\rangle\right)$, they have obtained for the noiseless case $\lambda<0$ for $g \leq 1$ and the system goes to the trivial fixed point $K^{*}=0$. For $g>1, \lambda>0$ and the system shows chaos.

They have numerically studied the effect of noise on chaotic properties of the system using the following form for $F(h)$ :

$$
F(h)= \begin{cases}-1 & \text { for } h<-1 / g, \\ g h & \text { for }-1 / g \leq h \leq+1 / g, \\ +1 & \text { for }+1 / g<h .\end{cases}
$$

For $g$ less than some critical value $g_{c}$, activity $K^{*}$ increases with noise as the system settles onto the stable fixed point $K^{*}=1+\sigma^{2}$, whereas for $g>g_{c}$ chaos sets in. It was found that for small noise asymptotically $g_{c}=$ $1-\sigma^{2} \ln \sigma^{2}$ and for large noise $g_{c}=\sqrt{\pi / 2} \sigma$. Fig. 1 is the plot of $g_{c}$ verses $\sigma$ (phase diagram), which clearly shows the chaotic and the regular regimes.

MSS concluded that for higher dimensional systems of this type where chaos occurs essentially due to the randomized interactions amongst constituent nonchaotic elements, noise acts to impair information flow between these elements and thereby suppresses chaos.

\subsection{Spatiotemporal stochastic resonance in excitable media}

Let us now consider the observation of stochastic resonance phenomenon in a spatially extended pattern forming system, as reported by Jung and Mayer-Kress (JMK) [41]. Stochastic resonance, as the name suggests, is a phenomenon in which at a given noise-strength certain temporal patterns 
of the system get enhanced drastically owing to an increase in the system's sensitivity [19, 20, 25]. This behaviour has a characteristic bell-shaped curve when the output power (of fourier spectrum) at the corresponding frequency is plotted against noise-strength, with the peak at the 'resonating' noise value. In order to observe this type of phenomenon in an extended system JMK considered a two-dimensional equidistant square array (with lattice constant $a$ ) of $N \times N$ noisy threshold devices $d(i, j), i, j=1, \cdots, N$ with the following properties: if the input $x_{t}(i, j)$ to the devices is below some threshold $b$, output $y_{t}(i, j)=0$; if $x_{t}(i, j)>b$ the device generates a spiky output of the form $y_{t}(i, j)=I_{0} \Theta(\dot{x}(i, j)) \delta\left(x_{t}(i, j)-b\right)$ and then goes into temporary hibernation for a refractory period $\Delta t_{r}$. The dynamics can be described by a linear Langevin equation for inputs $x_{t}(i, j)$ as

$$
\dot{x}(i, j)=-\gamma x(i, j)+\sqrt{\gamma \sigma} \eta_{t}(i, j),
$$

where $\gamma$ is a leakage constant which accounts for the thermal leakage due to interaction with the surroundings, $\eta_{t}(i, j)$ is a delta-correlated noise and $\sigma=\left\langle x_{t}(i, j)^{2}\right\rangle$. Eq. (12) on integration over $\Delta t$ gives rise to the discrete time dynamics

$$
x_{t+\Delta t}(i, j)=x_{t}(i, j) \exp (-\gamma \Delta t)+G_{t}(i, j),
$$

where $G_{t}(i, j)$ is a Gaussian random number with variance $\sigma_{G}=\sigma\left[1-e^{-2 \gamma \Delta t}\right]$.

Threshold devices are pulse-coupled, i.e., when an element $d(k, l)$ fires, it communicates with its surrounding elements $d(i, j)$ at a distance $r_{i j, k l}$ apart by contributing an amount $K \exp \left(-\beta r_{i j, k l}^{2} / a^{2}\right)$ to their inputs $x_{t}(i, j)$, where $K \rightarrow K / b$ is in dimensionless unit and $\beta$ is a dimensionless quantity describing the spatial coupling range. For large coupling $(K \geq \exp (\beta))$, these firing elements give rise to excitatory waves spreading through the array, such as spiral waves, target waves (single, nonrepetitive wavefronts) etc. The selection of the wave form depends on the geometry of initial conditions. There is a threshold $K_{c}$ such that for $K<K_{c}$ excitatory waves do not necessarily start.

However, in the presence of noise the spreading of excitatory waves, such as spiral waves, is observed in subthreshold regime $\left(K<K_{c}\right)$. Any such noise-sustained wave disappears soon after the noise is turned off. With increasing noise the spiral wave evolves with a larger curvature, i.e., with an enhanced coherence in the array. If the noise is increased further, the spiral breaks up and there is a loss of coherence. 
In order to show that the phenomenon observed above is essentially spatiotemporal stochastic resonance, JMK have taken a solitary initial wave and defined a quantity $\mu$, time averaged number of excess events, as the difference of number of firing elements along the driving wavefront and average number of firing events along a row of the array, not affected by the driving. The plot of $\mu$ versus $\sigma$, the variance of noise, is shown in figure 2. The plot exhibits

the characteristic bell-shaped curve mentioned earlier. Synchronization of the spatiotemporal firing pattern to an external driving, thereby giving rise to the resonating peak, is a simple generalization of the stochastic resonance to this extended system.

We shall now move on to the final part of the paper, effect of noise on coupled map lattices (CML). As the model itself has a plethora of interesting dynamical features, we begin with an introduction on CML.

\section{Coupled map lattice}

Coupled map lattices (CML) are spatially extended dynamical systems with discrete space (lattice), discrete time and continuous state evolution. General evolutionary dynamics of a CML may be expressed as

$$
x_{t+1}(i)=F\left(x_{t}(i)\right)+\sum_{j \neq i} \varepsilon_{i j} G\left(x_{t}(j), x_{t}(i)\right),
$$

where $F$ and $G$ are nonlinear maps and the state variables $x_{i}$ varies continuously in the phase space of the map and $i$ is the space index. CMLs are in general constructed with the symmetry properties of spatial translational and rotational invariance, and so the coupling term $\varepsilon_{i j}$ is taken to be a uniform scalar ranging from the nearest neighbour coupling to global coupling.

CMLs have been used to model many physical phenomena. These include pattern formation, chemical waves, excitable media, nucleation, crystal growth, charge density waves, population dynamics, fluid dynamics etc. [42-55]. Chemical systems exhibit very rich spatiotemporal structures and patterns. Standard description of these systems is in terms of the reactiondiffusion equations that incorporate combined effects of local nonlinear reaction dynamics and global diffusion of chemical species due to the concentration gradients in the system. Many of the averaged and even some detailed features of chemical patterns can be captured by a simple CML description 
of the dynamics. Another area of interest is the phase ordering dynamics. Phase separation occurs due to competition between different states in the system, such as domain growth in ferromagnetic and chemical systems. Some of the salient features of phase ordering dynamics are also observed in CML model. CML may also be applied in pattern dynamics with an excitable state and a relaxation from it. Such processes are observed in reaction-diffusion in excitable media and also in biological problems such as heart rhythm and electrical activities in neural tissues. In fluid dynamics, simulations using CML can show formation of convective patterns, vortices, sinks, sources etc. Here CML may offer a computationally economical way of simulating real behaviour. One of the major interests in studying CML is in the context of understanding the spatial and temporal structure formation, specially in fluid dynamics. These structures appear in spite of the fact that the underlying evolution of the system is spatiotemporally chaotic.

One of the most extensively studied types of one dimensional CML is that with nearest-neighbour coupling and having the following form:

$$
x_{t+1}(i)=F\left(x_{t}(i)\right)+\frac{\varepsilon}{2}\left[G\left(x_{t}(i-1)\right)+G\left(x_{t}(i+1)\right)-2 G\left(x_{t}(i)\right)\right] .
$$

We shall concentrate on the form $G(x)=F(x)$, which reduces Eq. (15) to

$$
x_{t+1}(i)=(1-\varepsilon) F\left(x_{t}(i)\right)+\frac{\varepsilon}{2}\left[F\left(x_{t}(i-1)\right)+F\left(x_{t}(i+1)\right)\right] .
$$

This particular model is known as future diffusive CML since the diffusively coupled entities are one timestep evolved values. Studies of this model alongwith the local dynamics as logistic map $F(x)=\mu x(1-x)$ have revealed that they can exhibit a wide range of spatiotemporal complexity. It has been observed that the temporal period doubling behaviour of the map can induce spatial domain structures separated by kinks and antikinks. A pattern selection regime is observed where patterns of certain characteristic lengths are selected. Phenomena such as spatiotemporal quasiperiodicity, soliton turbulence, spatiotemporal intermittency, wavelength doubling bifurcations, synchronization etc. for this and other maps (like circle map $\theta_{t+1}=\theta_{t}+\Omega-\frac{K}{2 \pi} \sin \left(2 \pi \theta_{t}\right), \Omega-$ angular frequency, $K$ - nonlinearity parameter) have been identified [56-64].

We now discuss the effect of noise on the evolution of CML. In the next subsection we report a novel phenomenon that we have observed in this context 65. 


\subsection{Noise and 'stochastic coherence' in CML}

We take the system of Eq. (16) with an additional noise term as follows:

$$
x_{t+1}(i)=(1-\varepsilon) F\left(x_{t}(i)\right)+\frac{\varepsilon}{2}\left[F\left(x_{t}(i-1)\right)+F\left(x_{t}(i+1)\right)\right]+\eta_{t},
$$

where $\eta_{t}$ is the familiar delta-correlated noise. Logistic function $F(x)=$ $\mu x(1-x)$ is used as local dynamics. For noise $\eta_{t}$ we have used a uniformly distributed random number bounded between $-W$ and $+W$, with the constraint that $0<x_{t+1}(i)<1$; we call $W$ the noise-strength parameter. Values of $\mu, \varepsilon$, and $L$ (size of the lattice) are chosen so that the resultant dynamics of the system is chaotic.

Now, we define a structure as a region of space such that the difference in the values of the variables of neighbouring sites within this region is less than a predefined small positive number say $\delta$, i.e., $\left|x_{t}(i)-x_{t}(i \pm 1)\right| \leq \delta$. We call $\delta$ the structure parameter. We look for such patterns, or structures, to appear in the course of evolution of the model given by Eq. (17).

Figure 3 shows a plot (on log-log scale) of the distribution of the number $n(l)$ vs. length $l$ of the structures for different values of $W$, with $\mu=4.0$, $\epsilon=0.6, \delta=0.0001$ and $L=1000$, and open-boundary conditions are used. Power-law nature of the decay of $n(l)$ is clearly evident, which has a form

$$
n(l) \propto l^{-\alpha},
$$

where $\alpha$ is the power-law exponent. This indicates that the system does not have any intrinsic length scale. It may be noted that in the absence of noise $(W=0.0)$ the decay is manifestly exponential [66]. Exponent $\alpha$ is seen to depend on the noise-strength $W$, with a minimum for $W$ around 0.6. We define average length $\bar{l}$ of a structure as $\bar{l}=\sum \ln (l) / \sum n(l)$. In Fig. 4 we plot the variation of $\bar{l}$ with $W$ for values of parameters as in Fig. 3. The plot exhibits a bell-shaped nature within a fairly narrow range of $W$ around value 0.6 (one may note the surprising similarity between figures 4 and 2, though they refer to completely different phenomena). It may be noted that the minimum of $\alpha$ also occurs for $W$ quite close to this value, as expected.

We call the phenomenon observed above stochastic coherence. This is similar to stochastic resonance which shows a bell-shaped behaviour of temporal response as a function of the noise-strength, as mentioned before. However one may note that our system does not have any intrinsic length-scale, 
whereas in stochastic resonance noise resonates with a given time-scale; hence our use of the word coherence rather than resonance. In stochastic resonance noise transfers energy to the system at a characteristic frequency, whereas in stochastic coherence noise induces coherence to the system.

To study the evolutionary aspects of these structures we obtained distribution of number $n(\tau)$ of structures vs. their lifetimes $\tau$ for different $W$. $n(\tau)$ is observed to decrease with $\tau$ with a stretched exponential type of decay having a form

$$
n(\tau) \propto \exp \left(-(\text { const. }) \tau^{\zeta}\right),
$$

where $\zeta$ depends on $W$. We define average lifetime $\bar{\tau}$ of a structure as $\bar{\tau}=$ $\sum \tau n(\tau) / \sum n(\tau)$. In Fig. 5 we plot $\bar{\tau}$ vs. $W$ for parameter values as in Fig. 3 . The graph shows a bell-shaped feature with maximum for $W$ around 0.6.

In order to ascertain the chaotic nature of the system evolution we have calculated the lyapunov exponent spectra for our system. We find a number of lyapunov exponents to be positive, implying that the underlying evolution is chaotic. Maximum lyapunov exponent $\lambda$ shows a minimum around noise-strength 0.6 The fact that we have observed other extrema for similar $W$ may make it appear that probably the origin of these behaviours lies with reduction of $\lambda$ due to noise [67] (though it should be noted that the reduction is not monotonic). To explore this possibility we have studied the variation of $\lambda$ with coupling parameter $\varepsilon$. We found that $\lambda$ remains fairly constant for $0.2 \leq \varepsilon \leq 0.8$ for the entire range of $W$. On the other hand, a plot of variation of average length $\bar{l}$ with $\varepsilon$ for fixed $W$ shows a monotonically increasing behaviour, quite contraty to what is expected from $\lambda$. This implies that the lyapunov exponent alone cannot be used for proper characterization of spatio-temporal features of the system (unlike the synchronization phenomenon discussed earlier, where $\lambda$ is sufficient to fully characterize the behaviour).

To conclude this section, we have reported a new phenomenon observed in a chaotically evolving one-dimensional CML driven by an identical noise, which we termed stochastic coherence. It is observed that there is a phenomenal increase in the abundance of coherent structures of all scales due to noise. Distribution of these structures shows a power-law decay with length of the structure. Average length as well as average lifetime of these structures exhibit characteristic maxima at certain noise-strength. 


\section{Summary and conclusion}

We have reviewed the work that has been carried out on the effect of noise on evolutionary dynamics of chaotic systems. In the case of uncoupled systems, we have encountered synchronization phenomenon for different phase space trajectories driven by identical noise above a certain strength. We have also seen nonstatistical behaviour for an ensemble of chaotic trajectories driven by an identical sequence of parametric noise. For coupled systems, noise above a given strength has been observed to suppress chaos in a random neural network with infinite range interactions. Spatiotemporal stochastic resonance, first case of stochastic resonance phenomenon observed in systems with spatial extension, has been reported for a two dimensional square array of firing devices. In the context of CML, we have observed an interesting phenomenon, stochastic coherence, when the entire lattice is driven by identical noise.

As noted earlier in the text, understanding formation and evolution of patterns and structures in the spatially extended systems is still incomplete. In real physical situations, noise almost ubiquitously influences the system behaviour. So while studying the ordering phenomena, incorporating noise as a part of the evolving 'supersystem' and studying mutual interaction of the two subsystems offers a more practical way of looking into the problem. The inherent nonlinearity in the system, which on one hand gives rise to chaos and on the other hand ordered behaviour, is surely again playing its role in enhancing order under the influence of noise; how so is still not quite very clear. We have encountered two kinds of noise induced ordering phenomena: global ordering (such as synchronization) and local ordering (spatiotemporal stochastic resonance and stochastic coherence). Stochastic coherence, in particular, may turn out to be a promising way towards achieving any degree of understanding of the structure formation in extended systems, although a lot of work remains to be done. While it is definitely not claimed that all

questions will be answered with this approach, we certainly hope to bridge a few gaps in the existing knowledge.

\section{Acknowledgments}

One of the authors (M.R.) acknowledges University Grants Commission (In- 
dia) and the other (R.E.A.) acknowledges Department of Science and Technology (India) for financial assistance. 


\section{References}

[1] R. M. May, Nature 261, 459 (1976).

[2] M. C. Mackey and L. Glass, Science 197, 287 (1977).

[3] A. Libchaber and J. Maurer, in Nonlinear Phenomena at Phase transitions and Instabilities (ed. T. Riste), 259 (1982).

[4] S. K. Scott, Chemical Chaos (1993), Oxford University Press, Walton Street, Oxford.

[5] P. Y. Cheumg and A. Y. Wong, Phys. Rev. Lett 59, 551 (1987).

[6] J. R. Tredicce and L. M. Narducci, in Directions in Chaos (ed. Hao Bai Lin), Vol 1, 148, World Scientific, Singapore (1987).

[7] P. S. Linsay, Phys. Rev. Lett. 47, 1349 (1981).

[8] J. O. Kephart, T. Hogg and B. A. Huberman, Phys. Rev. A 40, 404 (1989).

[9] T. Hogg, B. A. Huberman and M. Youssefmir, preprint (1995).

[10] Order And Chaos in Nonlinear Physical Systems, edited by S. Lundqvist, N. H. March and M. P. Tosi, Plenum Press, New York (1988).

[11] R. W. Schefer et.al., Phys. Fluids 6(2), 652 (1994).

[12] K. H. Park, R. W. Metcalf and F. Hussain, Phys. Fluids 6(2), 885 (1994).

[13] E. Stone and P. Holmes, Physica D 37, 20 (1989).

[14] S. D. Meyeres, J. Sommeria and H. L. Swinney, Physica D 37, 515 (1989).

[15] A. K. M. F. Hussain, Phys. Fluids 26, 2816 (1983).

[16] L. Sirovich, Physica D 37, 126 (1989).

[17] Topological Fluid Mechanics (ed. H. K. Moffatt et.al.), proceedings of the IUTAM symposium 1989, Cambridge University Press (1990). 
[18] Turbulence And Stochastic Processes: Kolmogorov's Ideas 50 Years On (ed. J. C. R. Hunt et.al.), The Royal Society, London (1991).

[19] R. Benzi, A. Sutera and A. Vulpiani, J. Phys. A 14, 1453 (1981).

[20] B. McNamara and K. Weisenfeld, Phys. Rev. A 39, 4854 (1989).

[21] L. Gammaitoni, F. Marchesoni, E. Manichella-Saetta and S. Santucci, Phys. Rev. Lett. 62, 349 (1989).

[22] Hu Gang, G. Nicolis and C. Nicolis, Phys. Rev. A 42, 2030 (1990).

[23] M. I. Dykman, R. Mannella, P. V. E. McClintock and N. G. Stocks, Phys. Rev. Lett. 65, 2606 (1990).

[24] J. Stat. Phys., edited by F. Moss, A. Bulsara and M. Shlesinger 70, $1-514$ (1993).

[25] R. F. Fox and Yan-nan Lu, Phys. Rev. E 48, 3390 (1993).

[26] A. R. Bulsara and L. Gammaitoni, Physics Today, 39 (1996).

[27] P. Jung, Phys. Rev. E 50, 2513 (1994).

[28] Noise in Dynamical Systems, edited by F. Moss and P. V. E. McClintock, Cambridge University Press, Cambridge, U.K. (1989), Vol 1-3.

[29] Noise and Chaos in Nonlinear Dynamical Systems, edited by F. Moss, L. A. Lugiato and W. Schleich, Cambridge University Press, Cambridge, U.K. (1990).

[30] D. A. Kurtze, Phys. Rev. Lett. 77, 63 (1996).

[31] R. J. Deissler, Phys. Lett. A 120, 334 (1987).

[32] H. R. Brand and R. J. Deissler, Phys. Lett. A 130, 293 (1988).

[33] A. Pikovsky, in Nonlinear and Turbulent Processes in Physics, edited by R. Z. Sagdeev (Harwood Acad., Chur, Switzerland, 1984); Phys. Lett. A 165, 33 (1992).

[34] S. Fahy and D. R. Hamann, Phys. Rev. Lett. 69, 761 (1992). 
[35] A. Maritan and J. R. Banavar, Phys. Rev. Lett. 72, 1451 (1994).

[36] A. Pikovsky, Phys. Rev. Lett. 73, 2931 (1994).

[37] A. Maritan and J. R. Banavar, Phys. Rev. Lett. 73, 2932 (1994).

[38] P. M. Gade and C. Basu (to be published in Phys. Lett. A).

[39] S. Sinha, Phys. Rev. Lett. 69, 3306 (1992).

[40] L. Molgedey, J. Schuchhardt and H. G. Schuster, Phys. Rev. Lett. 69, 3717 (1992).

[41] P. Jung and G. Mayer-Kress, Phys. Rev. Lett. bf 74, 2130 (1995).

[42] G. L. Oppo and R. Kapral, Phys. Rev. A 36, 5820 (1987).

[43] D. Kessler, H. Levine and W. N. Reynolds, Phys. Rev. A 42, 6125 (1990).

[44] Y. Oono and S. Puri, Phys. Rev. Lett. 58, 836 (1986).

[45] T. Yanagita and K. Kaneko, Phys. Lett. A 175, 415 (1993).

[46] M. H. Jensen, Phys. Rev. Lett. 62, 1361 (1989).

[47] R. V. Sole and J. Valls, Phys. Lett. A 153, 330 (1991).

[48] I. Waller and R. Kapral, Phys. Rev. A 30,2047 (1984).

[49] K. Kaneko, Prog. Theor. Phys. 74, 1033 (1985).

[50] R. J. Deissler and K. Kaneko, Phys. Lett. A 119, 397 (1987).

[51] F. H. Willeboordse and K. Kaneko, Phys. Rev. Lett. 73, 533 (1994).

[52] J. D. Keeler and J. D. Farmer, Phys. D 23, 413 (1986).

[53] S. Sinha, D. Biswas, M. Azam and S. V. Lawande, Phys. Rev. A 46, $6242(1992)$.

[54] I. Aranson, D. Golomb and H. Sompolinski, Phys. Rev. Lett. 68, 3495 (1992). 
[55] M. G. Cosenza and R. Kapral, Phys. Rev. A 46, 1850 (1992).

[56] J. P. Crutchfield and K. Kaneko, in Directions in chaos Vol 1, 272, World Scientific, Singapore (1987).

[57] Theory and applications of coupled map lattices, edited by K. Kaneko, John Wiley \& Sons, Chichester, England (1993).

[58] K. Kaneko, Phys. Lett. A 149, 105 (1990).

[59] H. Chate and P. Manneville, Phys. D 32, 409 (1988).

[60] R. E. Amritkar and P. M. Gade, Phys, Rev. Lett. 70, 3408 (1993).

[61] R. E. Amritkar, P. M. Gade, A. D. Gangal and V. M. Nandakumaran, Phys. Rev. A 44, 3407 (1991).

[62] P. M. Gade and R. E. Amritkar, Phys. Rev. E 47, 143 (1993).

[63] Qu Zhilin and Hu Gang, Phys. Rev. E 49, 1099 (1994).

[64] N. Chatterjee and N. M. Gupte, Phys. Rev. E 53, 4457 (1996).

[65] M. Roy and R. E. Amritkar (communicated to Phys. Rev. E).

[66] J. K. John and R. E. Amritkar, Phys. Rev. E 51, 5103 (1995).

[67] K. Matsumoto and I. Tsuda, J. Stat. Phys. 31, 87 (1983); 34, 111 (1984). 


\section{Figure Captions}

Fig. 1. Plot of phase diagram $\left(g_{c}\right.$ versus $\left.\sigma\right)$ obtained by Molgedey et.al. 40, for an infinite range neural network with external white noise. For a given gain $g$ chaos is suppressed for sufficiently strong noise $\sigma$.

Fig. 2. Plot of the graph of time averaged number of excess events $\mu$ with noise variance $\sigma$, for a typical lattice size $200 \times 200$ and with a given set of parameter values [11.

Fig. 3. Plot of variation of number $n(l)$ of structures with length $l$ for a lattice with size $L=1000$, for different values of noise-strength $W$ as indicated. Parameters chosen are $\varepsilon=0.6, \delta=0.0001$, and $\mu=4.0$. Open-boundary conditions are used. Data are obtained for 50000 iterates each for 10 initial condition.

Fig. 4. Plot of variation of average length $\bar{l}$ of structure with noise-strength $W$, with parameters as stated in Fig. 3 .

Fig. 5. Variation of average lifetime $\bar{\tau}$ of structures with noise-strength $W$ shown for parameters as stated in Fig. 3. 


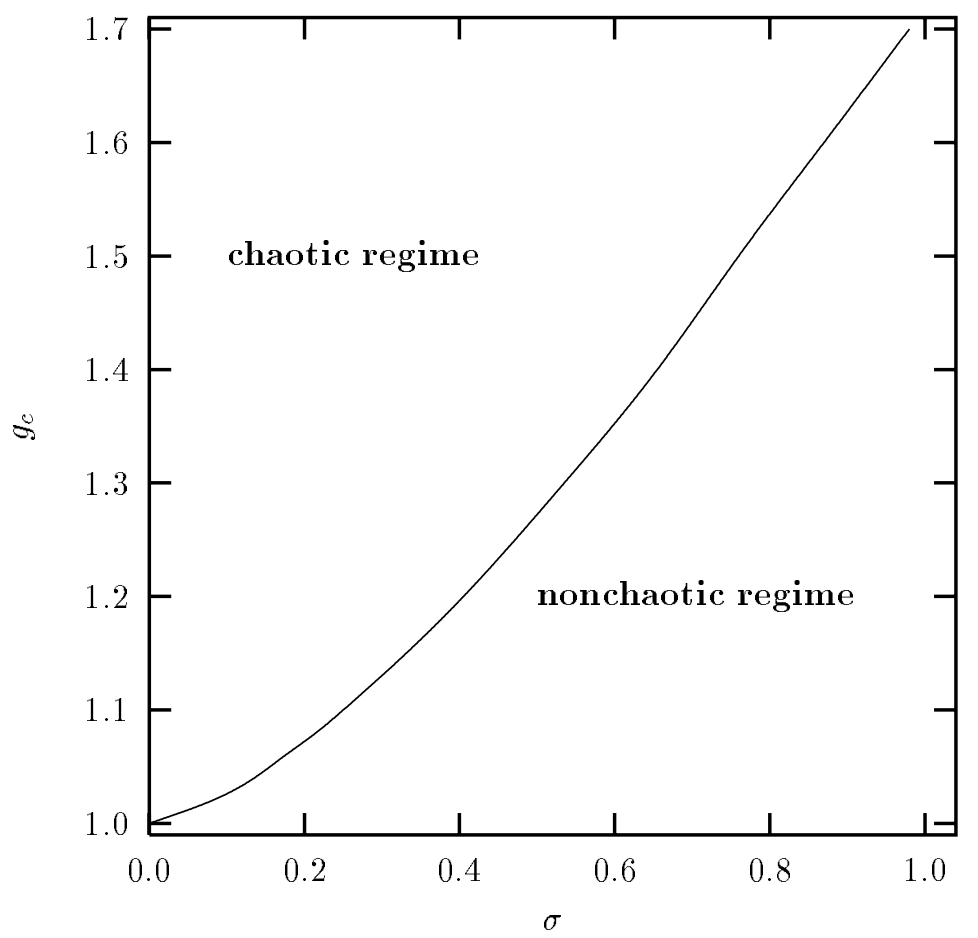

Fig. 1

MR \& REA 


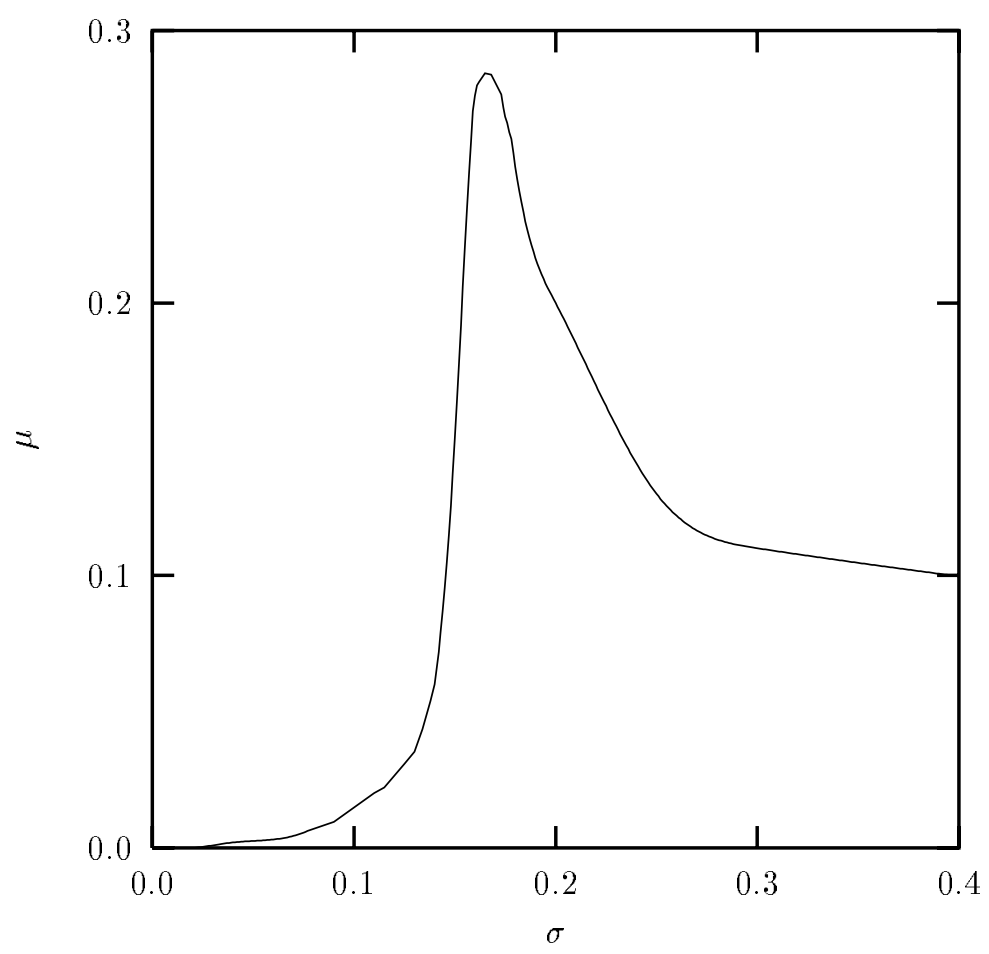

Fig. 2

MR \& REA 


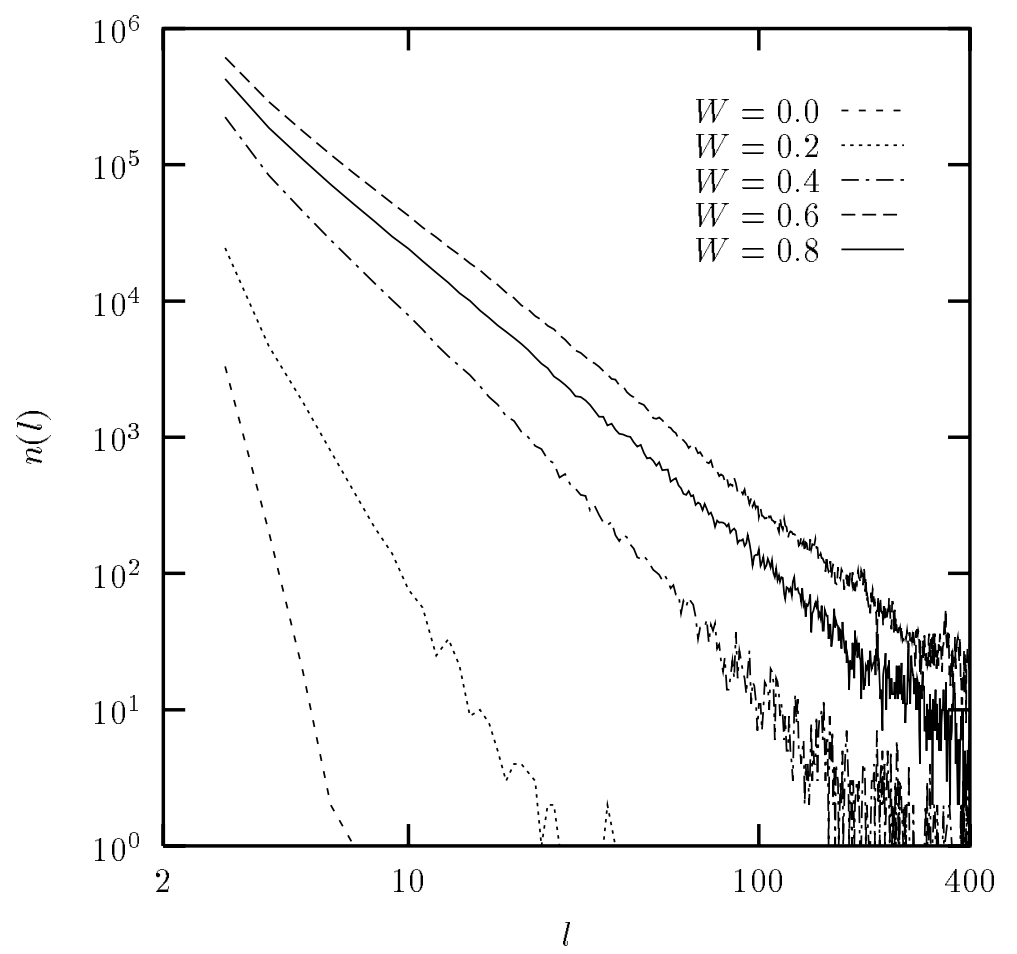

Fig. 3

MR \& REA 


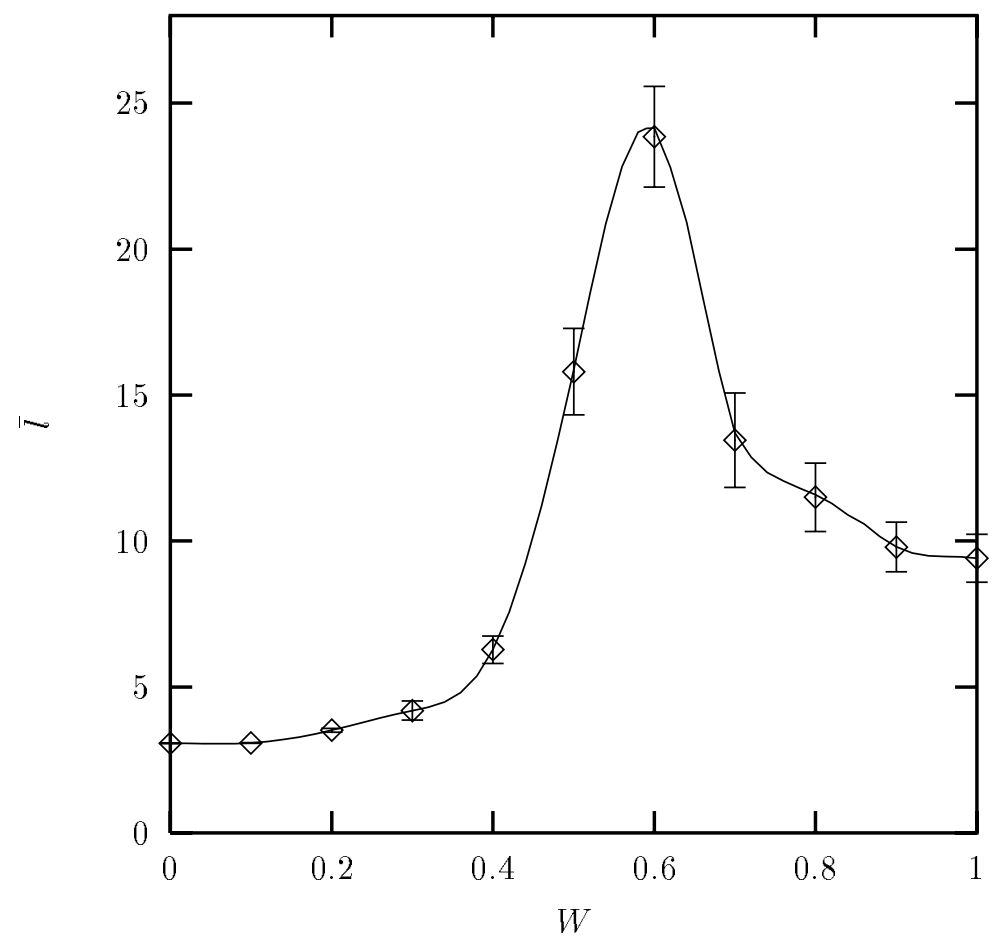

Fig. 4

MR \& REA 


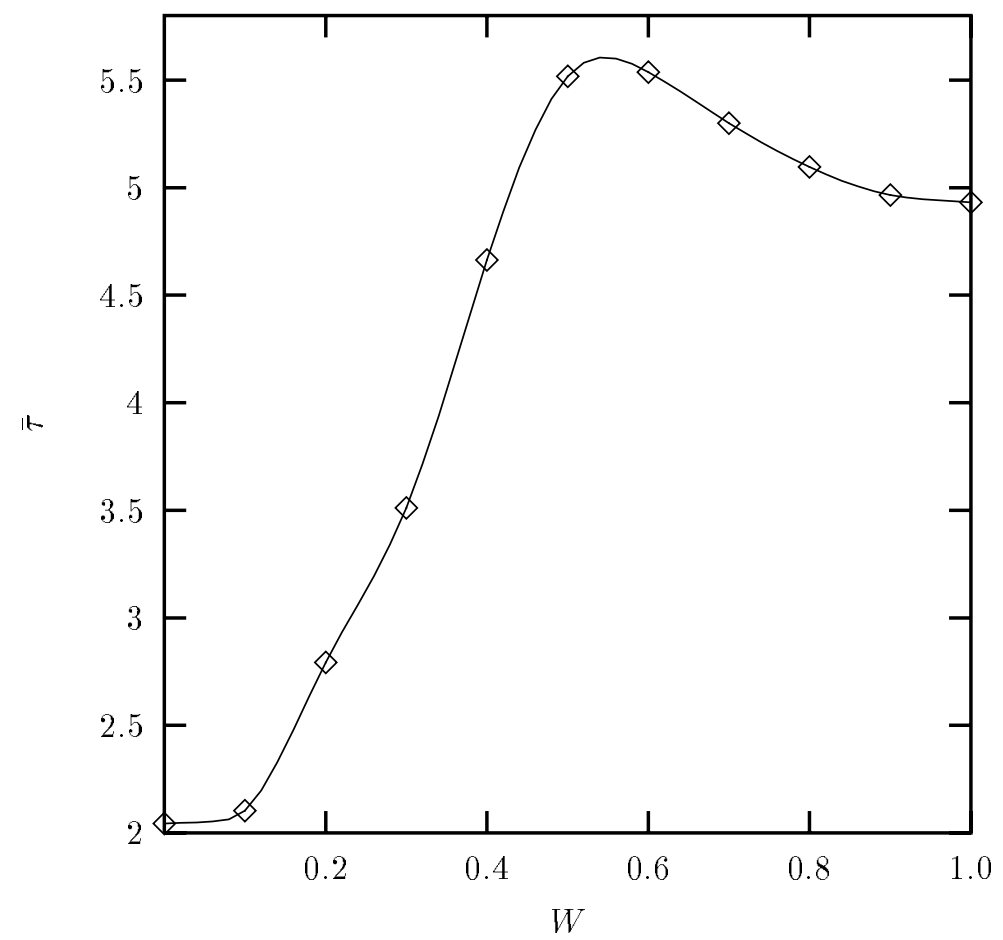

Fig. 5

MR \& REA 